\title{
Behavioral consequences of prion disease targeted to the hippocampus in a mouse model of scrapie
}

\author{
SAMAR BETMOUNI, ROBERT M. J. DEACON, J. NICHOLAS P. RAWLINS, and V. HUGH PERRY \\ CNS Inflammation Group, Oxford, England
}

\begin{abstract}
Sheep scrapie is an archetypal member of a group of chronic neurodegenerative diseases that can afflict both humans and animals and that are known collectively as the transmissible spongiform encephalopathies or prion diseases. The study of these diseases has been facilitated by the experimental transmission of sheep scrapie to laboratory rodents, and this has led to an ever-increasing literature on the pathogenesis and molecular biology of this unusual group of diseases. There is relatively little known about the relationship between the chronic neurodegenerative process and the development of clinical signs, the latter occurring very late in the course of the disease after a prolonged incubation period. We have previously shown that following intrahippocampal injection of scrapie brain homogenate, there is an early inflammatory response in the brains of affected mice. This develops long before there is neuronal loss and also before the mice develop any overt clinical signs of disease. It was surprising that despite the marked and evolving inflammatory response in the brains of scrapie-affected mice, indicative of underlying pathology, there were no outward signs that the mice had any form of central nervous system pathology. The present study shows that by using a number of behavioral tasks, including multitrial passive avoidance, open-field motor activity, and tests of muscle strength, it is possible to detect more subtle clinical signs much earlier during the course of disease in scrapie-affected mice. The relevance of this finding to the investigation of mouse scrapie is discussed.
\end{abstract}

Scrapie is a slow, progressive neurodegenerative disease that occurs naturally in sheep and goats. It is related to other transmissible spongiform encephalopathies such as Creutzfeldt-Jakob disease (CJD), Kuru, and bovine spongiform encephalopathy (BSE), which are also collectively known as the prion diseases. The central pathological event in these diseases is believed to be the conversion of a normal host protein (the prion protein, $\mathrm{PrPC}$ ) to one that is proposed to have a different conformation (Prusiner, 1982, 1996). This disease-associated protein is termed $\mathrm{PrPSc}^{\mathrm{Sc}}$ and is distinguishable from the normal protein by its resistance to a number of physical and chemical treatments and by the finding that it accumulates within disease-affected brains as insoluble deposits of amyloid. The prion diseases have a typical neuropathology of vacuolation, gliosis, and neuronal loss; in addition, it has long been held that they lack an inflammatory and an immune component (Prusiner, 1996). However, Betmouni, Perry, and Gordon (1996) have recently demonstrated that there

This work was funded by British Biotech Pharmaceuticals, Ltd., Cowley, Oxfordshire, England. R.M.J.D. and J.N.P.R. were supported by a Medical Research Council grant to the Interdisciplinary Research Centre for Cognitive Neuroscience. The authors would also like to thank John Harris and Peter Flaxman (Department of Pharmacology, Oxford) for their technical support in the making of the passive avoidance apparatus. Correspondence should be addressed to S. Betmouni, CNS Inflammation Group, School of Biological Sciences, University of Southamptom, Southampton S016 7PX, England. is an early inflammatory response in the brains of scrapieaffected mice, as shown by the presence of activated microglia and cytotoxic T-cells. This response, indicative of some neuronal pathology, was first seen at 8 weeks after intracerebral (i.c.) injection of scrapie-affected brain homogenate, long before any overt clinical signs of scrapie. The typical clinical signs of mouse scrapie were first observed at 23 weeks postinjection and consisted of a reduction in mobility, hunched posture, and poor coat condition. More advanced clinical signs occurred between 24 and 27 weeks and were typified by weight loss and a profound reduction in the mobility of the mice.

It is often assumed that the clinical signs of the prion diseases are secondary to neuronal loss, although some evidence suggests that the vacuolar change seen in these diseases does not always correlate with the severity of the clinical signs (Hadlow, Kennedy, \& Race, 1982). Our previous work has shown that in scrapie-affected mice, the inflammatory response with activated microglia (from 8 weeks postinjection) precedes the neuronal loss (from 20 weeks postinjection), which in turn precedes the appearance of clinical signs of disease (from 23 weeks postinjection). The presence of activated microglia is known to be a very sensitive indicator of the most subtle nervous system pathology (Kreutzberg, 1996). Since the prion agent ME7 is known to target the hippocampus (Fraser \& Dickinson, 1973), and the consequences of hippocampal lesions in rodents have been well studied (Gray \& McNaughton, 1983), we initiated the lesions in this structure 

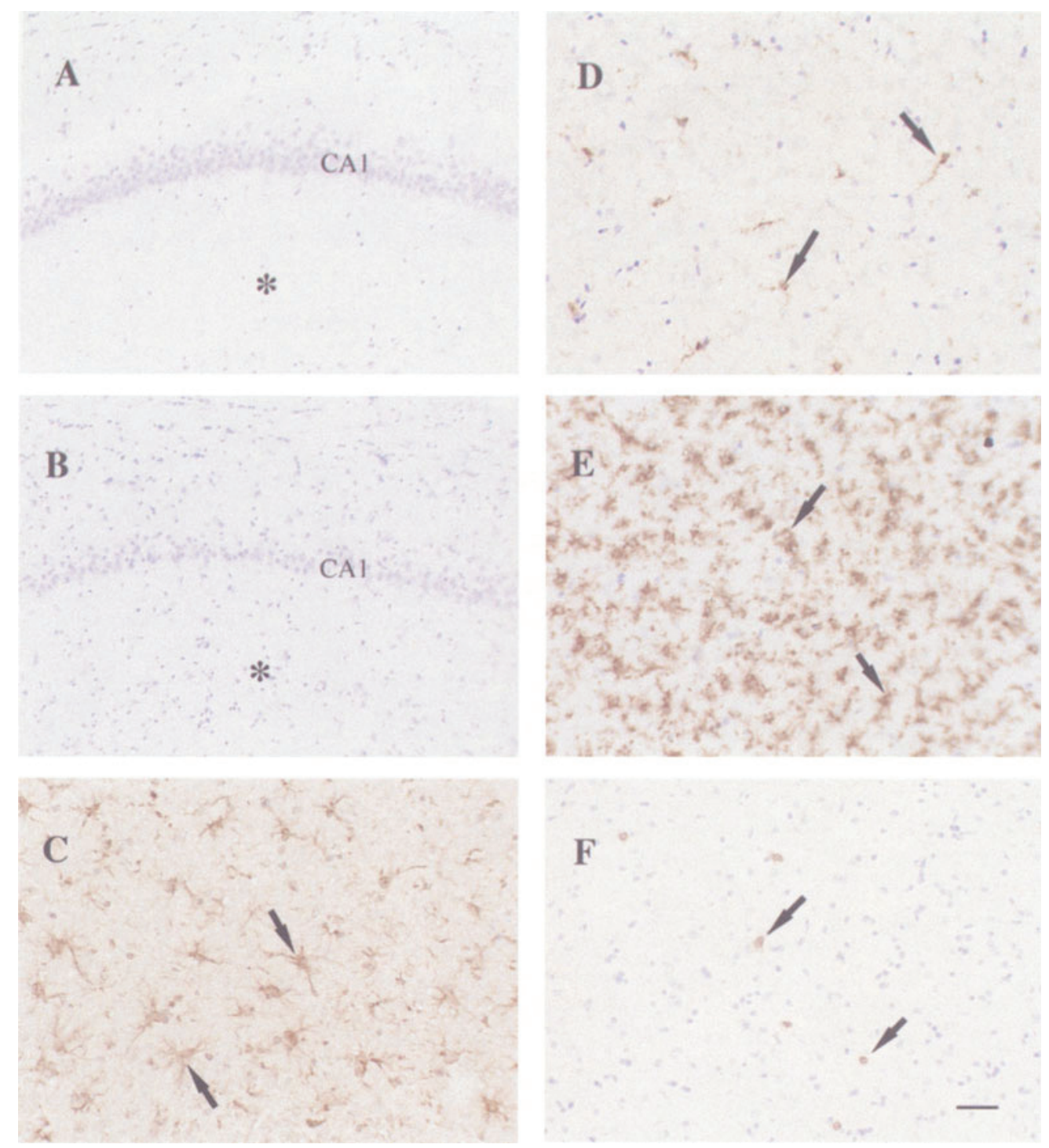

Figure 1. Histological examination of brain sections taken from mice with advanced clinical signs of scrapie following intracerebral injection of ME7 and compared with brain sections from the brains of age-matched mice previously injected with normal brain homogenate. (A) Photograph showing cresyl violet staining of CA1 region of the dorsal hippocampus in a mouse previously injected with normal brain homogenate. Note the normal appearance and thickness of the pyramidal layer of the CA1 and compare with the reduced thickness of the CA1 layer in a mouse with advanced clinical signs of scrapie (B). Note also the increased cellularity of the surrounding brain parenchyma (marked by an asterisk) in the brain of a mouse with advanced clinical signs of scrapie (B) compared with the normal appearance of the CA1 region and surrounding parenchyma in (A). (C) Photograph showing the typical astrocytosis characteristic of mouse scrapie. The astrocytes, marked by arrows, have been visualized using an antibody against glial fibrillarly acidic protein (GFAP). (D) Photograph showing the appearance of normal resting microglia in a mouse injected with normal brain homogenate. Compare this with the appearance of activated microglia in a brain section taken from a mouse injected with ME7 (E). The microglia (marked by arrows) were visualized using an antibody directed against macrosialin (FA11)-a membrane-associated endosomal component in macrophages. (F) A consecutive section of brain showing the distribution of CD3+ T-lymphocytes in an ME7-injected mouse. Scale bar $=50 \mu \mathrm{m}$.

using stereotaxic injections. We were interested to learn whether an early behavioral deficit could be detected if the appropriate test battery was deployed.

The behavioral tests were designed to examine the putative effects of scrapie injections on learning, memory, locomotor activity, and muscular strength and coordination. Evidence from earlier studies suggests that these aspects of behavior might be affected in the mouse scrapie model (Heitzman \& Corp, 1968; Hunter, Caulfield, \& Kimberlin, 1986; Lysons \& Woollard, 1996; McFarland 
\& Hotchin, 1980; Suckling, Bateman, Waldron, Webb, \& Kimberlin, 1976).

Previous reports of ataxia, abnormal posture, and tremors at or near the end of the incubation period have relied on simple observational techniques (Hunter et al., 1986). In our third experiment, we tested motor function and coordination more systematically with an apparatus widely used in psychopharmacological assessments of motor ability in order to characterize more fully the motor deficit.

\section{GENERAL METHOD}

\section{Subjects}

Eight-week-old male and female (Experiment 2) or male only (Experiments 1 and 3) C57BL/6J mice were housed in groups of $4-6$ in plastic cages in a temperature-controlled $\left(21^{\circ} \mathrm{C}\right)$ room on a 12:12-h light:dark cycle (lights on at $0800 \mathrm{~h}$ ). All testing was carried out during the light phase.

\section{Apparatus}

The passive avoidance apparatus was a rectangular box constructed of gray plastic, $24 \times 10 \times 29 \mathrm{~cm}$. One half of the floor consisted of a "safe" plastic side $(12 \times 10 \mathrm{~cm})$. The other side was a grid made from stainless steel bars of 3-mm diameter spaced $1 \mathrm{~cm}$ apart on the centers. Mild electric footshocks $(0.03 \mathrm{~mA}, 1.2$-sec duration) could be delivered to this grid by a constant current shock source and scrambler unit (Campden Instruments 521C and 521S).

Locomotor activity was measured in a clear plastic box $(31 \times 17$ $\times 21 \mathrm{~cm})$ marked off into rectangles $(10 \times 8.5 \mathrm{~cm})$. The invertible screen was a $30-\mathrm{cm}$ square of wire mesh consisting of $12-\mathrm{mm}$ squares of 1 -mm-diameter wire. The horizontal bar was a $2-\mathrm{mm}$-diameter steel rod, $26 \mathrm{~cm}$ long, held $20 \mathrm{~cm}$ above bench level by a vertical support (surfaced with wire mesh to provide a grip) at each end.

\section{Procedures}

All procedures were carried out in accordance with the requirements of the Animals (Scientific Procedures) Act of 1986.

\section{Passive Avoidance}

Each mouse was placed on the safe side of the apparatus, and the time taken for it to place all four feet on the grid was measured (initial latency). One footshock was delivered every $5 \mathrm{sec}$ that the mouse remained on the grid or whenever it returned to the grid (having crossed to the safe side). The criterion of avoidance learning was to remain for $60 \mathrm{sec}$ continuously on the safe side, after which the mouse was removed. All mice would successfully escape shocks (i.e., return to the safe side) after one (occasionally more) shocks. Most would then return to the grid side on several subsequent occasions before they learned to stay on the safe side for the requisite 60 sec. A maximum of 50 shocks was allowed; only 1 mouse (given scopolamine $0.5 \mathrm{mg} / \mathrm{kg}$ in Experiment 1) reached this maximum.

For retention testing (Experiments 2 and 3), the avoidance criterion was again for the mouse to remain on the safe side for $60 \mathrm{sec}$. If it did not do so, the procedure at acquisition was followed exactly as before, except now typically far fewer shocks were needed as a "reminder" treatment to achieve learning criterion.

In Experiment 1, scopolamine hydrobromide was injected intraperitoneally (i.p.) $15 \mathrm{~min}$ pretest in a volume of $10 \mathrm{ml} / \mathrm{kg}$. The vehicle (and control injection) was $0.9 \%$ saline.

\section{Locomotor Activity}

For the activity test, a mouse was placed in the activity box, and the number of rectangles crossed in 3 min was measured.

\section{Inverted Screen}

The inverted-screen test consisted of placing a mouse upright on the center of the screen, starting a stopwatch, and rotating the screen $180^{\circ}$ to an inverted position over a period of approximately $2 \mathrm{sec}$, the head of the mouse declining first. The mesh was held $20-30 \mathrm{~cm}$ above a sawdust-covered surface, and the time before the mouse fell off the mesh was recorded, up to a maximum of $60 \mathrm{sec}$. A score was assigned as follows: falling off between 0 and $10 \mathrm{sec}=1 ; 11$ and $25 \mathrm{sec}=2 ; 26$ and $60 \mathrm{sec}=3 ;>60 \mathrm{sec}=4$.

\section{Horizontal Bar}

For the horizontal-bar test, a mouse was held by the tail and lowered toward the center of the bar until it grasped it with both forepaws; then the tail was released and a stopwatch started. The time was measured until the mouse either fell off the bar or managed to move along it and touch one of the supports with one forepaw. To increase the reliability of assessing poor performers and minimize the influence of the experimenter's placement, if the mouse fell before $6 \mathrm{sec}$, it was retested up to two times to see whether it was capable of attaining a higher score. Scores on the bar were assigned a rank value as follows: falling off between 1 and $5 \mathrm{sec}=1 ; 6$ and 10 $\sec =2 ; 11$ and $20 \mathrm{sec}=3 ; 21$ and $30 \mathrm{sec}=4$; after 30 sec or reaching a bar support $=5$. For multiple tests, the mean rank value was calculated; thus a mouse falling off at $3 \mathrm{sec}$ followed by $8 \mathrm{sec}$ on retest would score 1.5 .

\section{Surgery}

The mice were anesthetized with i.p. Avertin $(0.1 \mathrm{ml} / 5 \mathrm{~g}$ body weight) and positioned with thẹir heads in a horizontal plane in a stereotaxic frame (Kopf Instruments). Then, $1 \mu \mathrm{l}$ of $10 \% \mathrm{w} / \mathrm{v}$ of ME7 brain homogenate, derived from the brain of a C57BL/6J mouse with clinical signs of scrapie, was injected bilaterally into the dorsal hippocampus via a pulled-glass micropipette (coordinates from bregma: anterior-posterior -2.5 , lateral \pm 1.7 , ventral $-1.6 \mathrm{~mm}$ from the surface of the brain). Control mice were injected with $10 \%$ w/v normal brain homogenate derived from a normal C57BL/6J mouse. The mice were all housed in a Scantainer Type D (Scanbur Ltd., Denmark) and observed regularly.

\section{Histology}

At the end of the experiment, the mice were deeply anesthetized and perfused with $0.9 \%$ saline via the transcardiac route. The brains were removed, embedded in Tissue Tec (OCT embedding compound, Miles, Inc.), and frozen in isopentane on dry ice. Then, $10-\mu \mathrm{m}$ coronal sections were cut on a cryostat and processed for immunocytochemistry. The sections were fixed in absolute alcohol at $4^{\circ} \mathrm{C}$ for 10 min; the following primary antibodies were used: FAll to detect macrosialin (CD68), a lysosomal antigen in macrophages (Rabinowitz \& Gordon, 1991); KT3 to detect CD3, a pan T-cell marker (Tomonari, 1988); and an antiserum to glial fibrillary acidic protein (GFAP) to detect astrocytes (DAKO). The primary antibodies were detected by the avidin-biotin peroxidase method (Vectastatin Elite $\mathrm{ABC}$, Vector Laboratories), with diaminobenzidine as the chromogen (Lawson, Perry, Dri, \& Gordon, 1990).

\section{Experiment 1}

Since passive avoidance is generally performed using a singletrial procedure, the sensitivity of the present multitrial technique and apparatus was first assessed by studying the effect of the anticholinergic drug scopolamine on the acquisition process (Deacon, 1990). The procedure was otherwise the same as that used for the scrapie investigations, with the exception that retention was not tested ( $n=10$ mice per group except for $n=11$ at the $0.5-\mathrm{mg} / \mathrm{kg}$ dose). In more recent work, Deacon (1998) has observed in many replications that retention is decreased in mice treated with scopolamine at acquisition. 


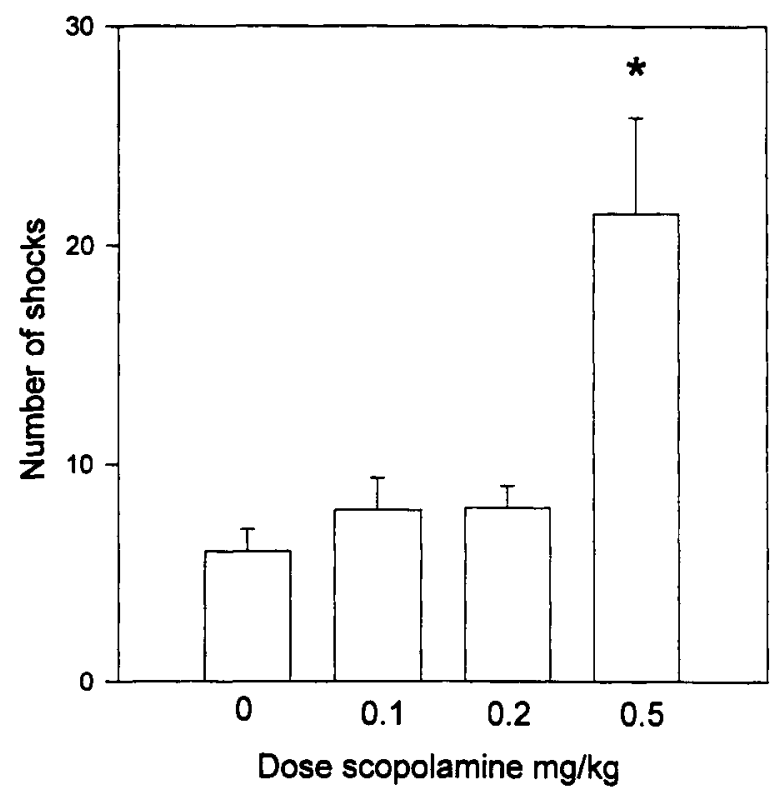

Figure 2. Experiment 1: The effect of scopolamine on the number of shocks to criterion for acquisition of the passive avoidance task. Mean $+S E M ;{ }^{*} p<.05$, Dunnett's test; $n=10$ per group except scopolamine $0.5 \mathrm{mg} / \mathrm{kg}(n=11)$. N.B.: Retention was not tested in this experiment.

\section{Experiment 2}

Acquisition of passive avoidance was conducted 8 weeks after injection with ME7 or normal brain homogenate. Both male and female mice were used ( $n=4$ mice per group, except for 6 female controls). Activity testing took place on the following day. Subsequent retention and activity testing occurred every 2 weeks (activity data for 22 weeks were not available).

\section{Experiment 3}

This experiment used only male mice because the results of Experiment 2 suggested that i.c. injection of ME7 brain homogenate had clearer effects in males. We increased the number of mice per group ( $n=10$ control; $n=10$ scrapie mice), but there was fighting and premature mortality in one scrapie cage, so early data from these mice ( $n=4$ ) were generally retrospectively excluded from analysis. Thus we did not achieve our target $n$ values. The inverted-screen and horizontal-bar tests of muscular function were performed from Week 12 (on the same day and after the passive avoidance retention tests).

\section{Statistics}

Experiment 1. Initial latency: A one-way analysis of variance (ANOVA) on ranks (Kruskal-Wallis test) was followed by Dunn's test on treated versus control mice. Shock: a one-way ANOVA of the number of shocks to criterion (cutoff $n=50$ ) was followed by Dunnett's test.

Experiment 2. A two-way ANOVA was conducted on the number of shocks to the passive avoidance criterion. Retention was assessed by comparing (within sexes) the proportion of scrapie and control mice failing to avoid the grid side of the apparatus over the weeks of testing (two-tailed $t$ test). Activity data were subjected to a two-way ANOVA followed by two-tailed $t$ tests for within-sex comparisons.

Experiment 3. Passive avoidance and activity data were analyzed as above; since the scores from the inverted-screen and horizontalbar tests were not normally distributed, the Mann-Whitney $U$ test (two-tailed) was used to compare scrapie and control mice. An alpha value of $p<.05$ was accepted as significant in all analyses.

\section{GENERAL RESULTS}

\section{Clinical Signs and Pathology}

Male and female mice developed the typical pathological changes (Figure 1) and clinical signs of scrapie at 24 weeks postinjection with ME7 brain homogenate. Mice injected with normal brain homogenate remained clinically well and did not differ from uninjected age-matched mice.

\section{Experiment 1}

Scopolamine significantly increased the initial latency to cross to the grid side [i.e., before the first shock; $\mathrm{H}(3)=$ $13.4, p<.0038]$. The difference between the $0.5-\mathrm{mg} / \mathrm{kg}$ dose and control mice was significant at the .05 level (Dunn's test). Scopolamine significantly increased the number of shocks required to reach the passive avoidance acquisition criterion at the $0.5-\mathrm{mg} / \mathrm{kg}$ dose $[F(3,37)=$ $7.79, p=.0003$; control vs. $0.5 \mathrm{mg} / \mathrm{kg}, p<.05$, Dunnett's test; see Figure 2]. No other doses were significant.

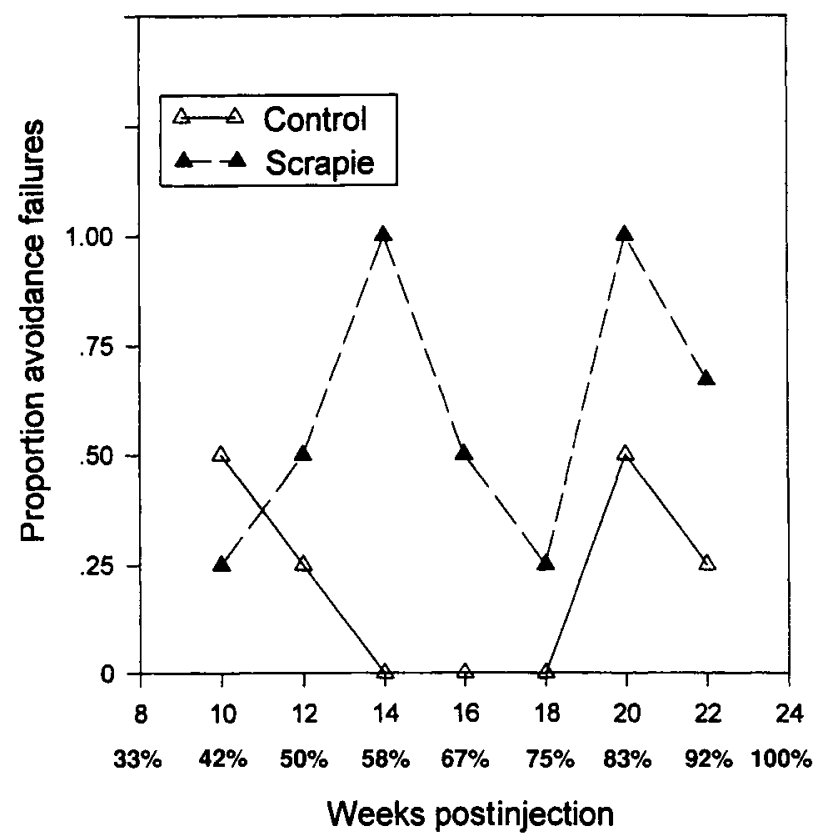

Figure 3. Experiment 2: Proportion of avoidance failures on the passive avoidance task in male mice ( $n=4$ per group) at each of the indicated times after intracerebral injection with either normal brain homogenate (control) or ME7 brain homogenate (scrapie). It can be seen that during the retention period (i.e., from 10 weeks onward), the proportion of mice failing to avoid was significantly greater in the ME7-injected group than in the mice injected with normal brain homogenate. N.B.: In Figures 3-10, the percentage incubation period is calculated on the assumption that unequivocal systemic clinical signs of scrapie occur at 24 weeks postinjection. 


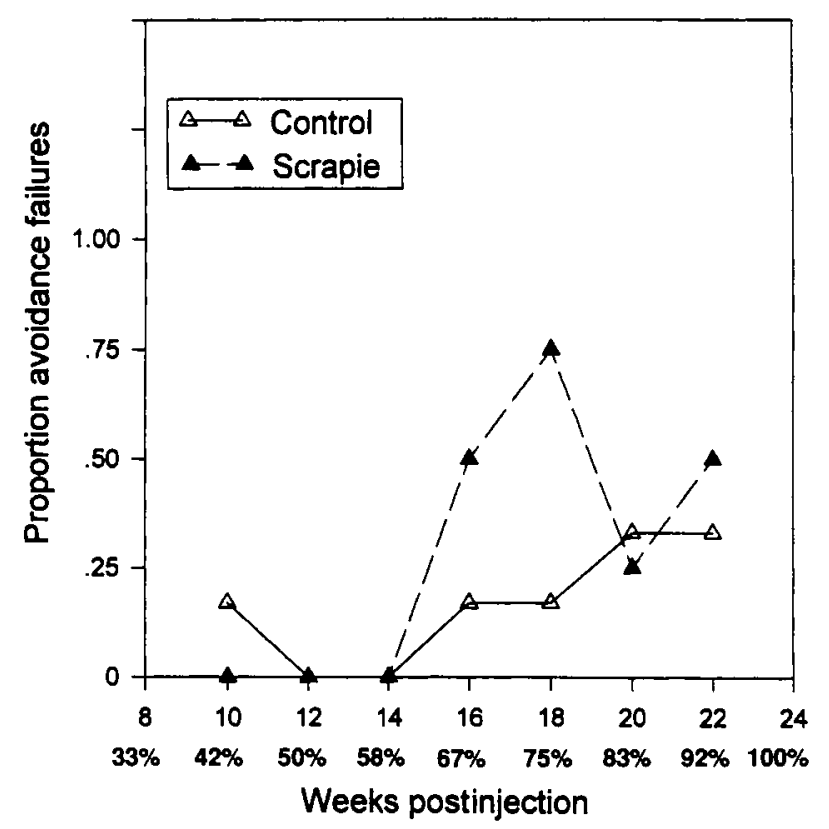

Figure 4. Experiment 2: Proportion of avoidance failures on the passive avoidance task in female mice $(n=6$ control, 4 scrapie) at each of the indicated times after intracerebral injection with either normal brain homogenate (control) or ME7 brain homogenate (scrapie). The increase in proportion of ME7injected female mice failing on the passive avoidance task occurred at a later time point in the course of the disease (from 16 to 18 weeks) than in male mice (Figure 3 ), but did not reach statistical significance.

\section{Experiment 2}

A two-way ANOVA (sex $\times$ group) revealed no significant difference in the median number of shocks to reach the passive avoidance acquisition criterion [sex, $F(1,14)=$ 3.73 ; group, $F(1,14)=1.15$; sex $\times$ group, $F(1,14)=$ 3.73]. There were no significant differences in the initial latency [sex, $F(1,15)=.375$; group, $F(1,15)=0.669$; sex $\times$ group, $F(1,15)=1.096]$. During retention testing, the proportion of mice failing to avoid was greater in the scrapie groups; a two-way ANOVA indicated a nonsignificant trend toward a sex difference [sex, $F(1,24)=$ 3.44 ; group, $F(1,24)=6.75$; sex $\times$ group, $F(1,24)=$ $1.87]$. The proportion failing to avoid was significantly greater in the males [unpaired $t$ test: $t(12)=2.62, p=$ .02 ; see Figures 3 and 4$]$. There was an increase in activity from 12 weeks after i.c. injection with ME7 brain homogenate, particularly in the males, followed eventually by lower activity and increased variability as clinical signs started to appear in some animals $[F(1,14)=5.158$, $p<.05$; see Figures 5 and 6$]$.

\section{Experiment 3}

As in Experiment 2, there was no significant difference in the initial latency or the number of shocks to the passive avoidance criterion [both $t \mathrm{~s}(18)<1.5$ ]. Scrapieaffected mice again showed poorer passive avoidance re- tention performance, replicating the effect found in Experiment 2 (Figure 7).

An overall ANOVA of the proportion of mice failing to avoid on each retention session, with data from Experiments 2 and 3 combined, showed a significant effect of treatment $[F(1,24)=7.83, p<.01]$, but not of replication $[F(1,24)=.07]$, and there was no significant interaction between these factors $[F(1,24)=1.29]$. The increase in locomotor activity was replicated, but occurred slightly later than in Experiment 2 (Figure 8). Muscle function was significantly impaired from Week 20 (Figures 9 and 10 ), before the conventional clinical signs of mouse scrapie were apparent at 24 weeks postinjection.

An additional analysis of the median number of shocks per criterion throughout retention (excluding acquisition) pooled data from Experiments 2 and 3. ME7-injected mice required slightly more shocks (median $=2$ ) than did controls (median $=1$ ) to reachieve the criterion of $60 \mathrm{sec}$ on the safe side (Mann-Whitney $U$ test: $\mathrm{W}=851, p=$ .0169 ). The median value for acquisition ranged from 3 to 8.5 , so considerably fewer shocks were required to achieve the $60-\mathrm{sec}$ criterion at retention than at acquisition.

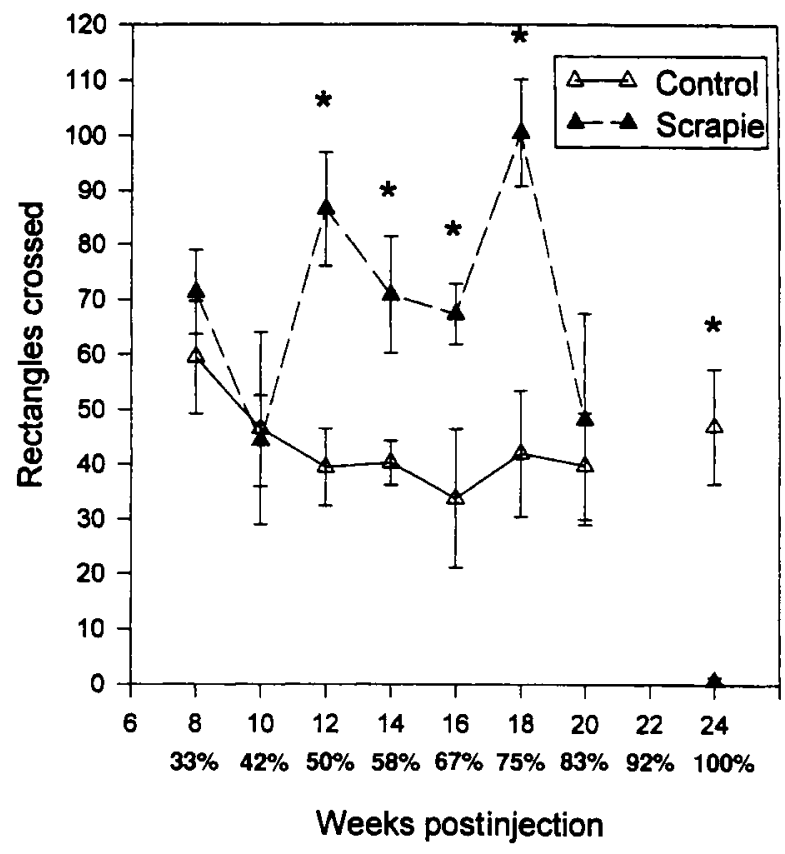

Figure 5. Experiment 2: Activity (mean $\pm S E M$ ) of male mice ( $n=4$ per group) injected intracerebrally with either ME7 (scrapie) or normal brain homogenate (control) at each of the indicated time points. The activity was measured as the number of rectangles crossed over a $3-\mathrm{min}$ period. It can be seen that there is a statistically significant increase in the activity of ME7injected mice (between 12 and 18 weeks) over that of mice injected with normal brain homogenate. The motor activity of ME7-injected mice began to decline from 18 weeks onward, and by 24 weeks it was significantly below that observed in control mice. ${ }^{\star} p<.05, t$ test, two-tailed. 


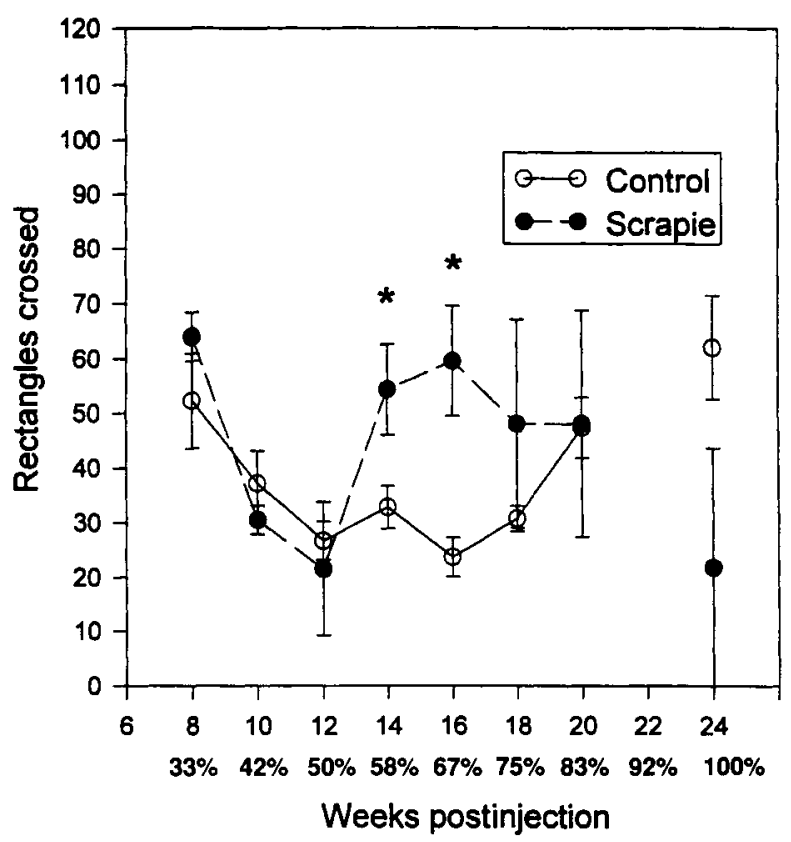

Figure 6. Experiment 2: Activity (mean $\pm S E M$ ) of female mice ( $n=6$ controls, 4 scrapie) injected intracerebrally with either ME7 (scrapie) or normal brain homogenate (control) at each of the indicated time points. The activity was measured as the number of rectangles crossed over a 3-min period. It can be seen that there was a statistically significant increase in the activity of ME7-injected mice (at 14 and 16 weeks) over that of mice injected with normal brain homogenate. The motor activity of the ME7injected mice began to decline from 16 weeks onward, and by 24 weeks it was lower than that seen in control mice, although this did not reach statistical significance. ${ }^{*} p<.05, t$ test, two-tailed.

Finally, a Mann-Whitney $U$ test combined data from Experiments 2 and 3 on the number of shocks received on each retention session. This showed that scrapie mice required significantly more shocks per retention session than did controls (medians $=2.0$ and 1.0 , respectively; $\mathrm{W}=851, p=.0169$ ).

\section{GENERAL DISCUSSION}

In this model, we have shown that it is possible to detect behavioral changes in scrapie-affected mice long before the conventional clinical signs of scrapie are apparent at 24 weeks postinjection. There were no effects on passive avoidance or locomotor activity at 8 weeks after injection with either normal or ME7 brain homogenate. This was followed by behavioral changes in the ME7injected mice; locomotor activity was increased at 12-14 weeks, and retention of the multitrial passive avoidance was impaired at 14 weeks postinjection. In the later stages of the disease, general systemic effects were observed in addition: Motor function was impaired both on the inverted-screen and on the horizontal-bar tests. It should be noted that although the impairment of muscle function was a late feature of the disease, it was still seen be- fore the conventional clinical signs of mouse scrapie were obvious some 4 weeks later.

The few temporal studies of behavioral changes in scrapie-affected mice have reported conflicting results (Heitzman \& Corp, 1968; Hunter et al., 1986; Lysons \& Woollard, 1996; McFarland \& Hotchin, 1980; Outram, 1972; Suckling et al., 1976). The variability between and within these studies may be attributable to factors such as the strains of mice, and the scrapie agent and the duration of incubation, in addition to the usual variables common to all behavioral work, such as apparatus and procedure. It is not possible to make direct comparisons between our results and the results discussed above for a number of reasons. All of the studies outlined above involved the injection of scrapie brain homogenate either systemically or into an ill-defined region of the brain. However, despite the nonspecific localization of the injection, Outram (1972) observed a decline in drinking as early as 7 weeks after i.c. injection of ME7 in C57BL/6J mice.

In the present studies, the ME7 strain was injected bilaterally into the dorsal hippocampus to produce a more targeted lesion. Several factors may account for the fact that the earliest behavioral change (increased motor activity) we detected resembled those seen following hippocampal damage: our use of an intrahippocampal focal

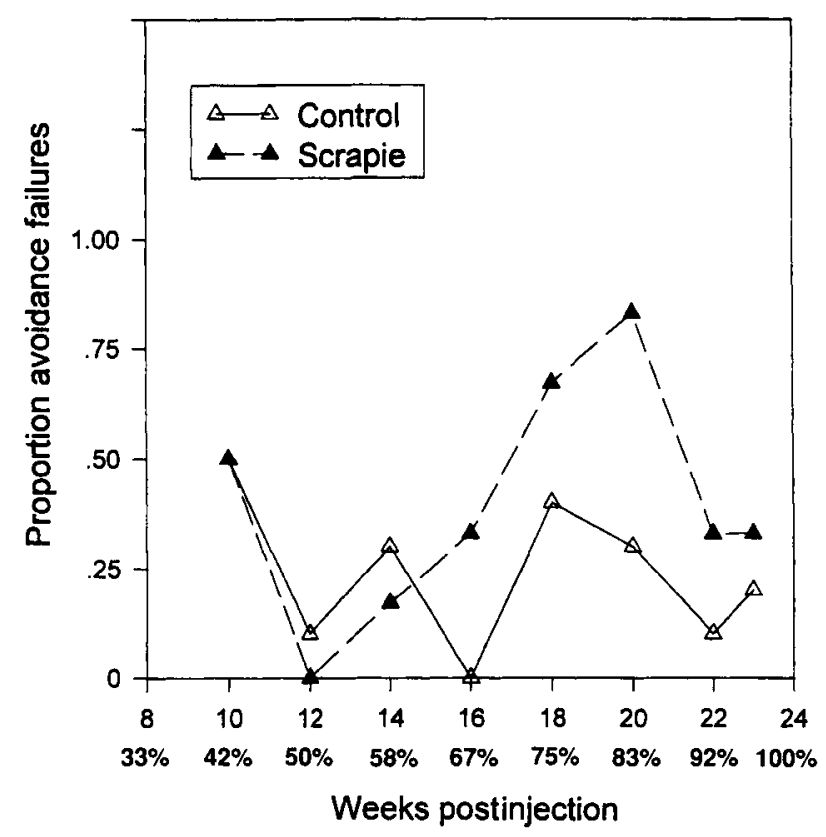

Figure 7. Experiment 3: Proportion of avoidance failures on the passive avoidance task, during the retention period, in male mice ( $n=10$ control, 6 serapie) at each of the indicated times after intracerebral injection with either normal brain homogenate (control) or ME7 brain homogenate (scrapie). There was a tendency for ME7-injected mice to show a poorer performance on the passive avoidance task, but this did not reach statistical significance. N.B.: The number of scrapic mice is low because of fighting-induced mortality. 


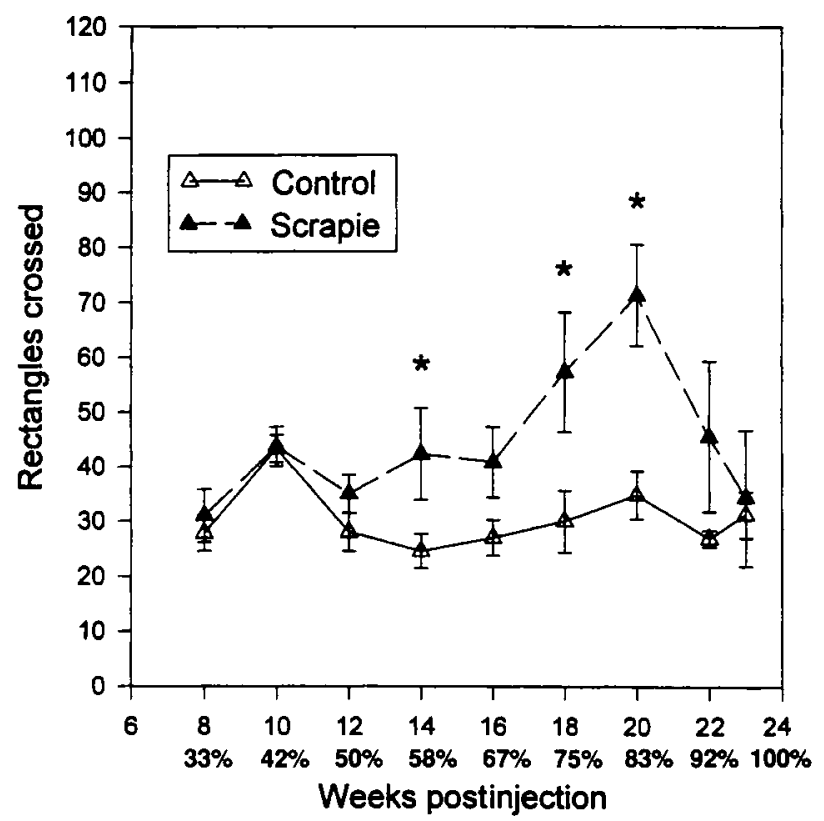

Figure 8. Experiment 3: Activity (mean $\pm S E M$ ) of male mice $(n=10$ control, 6 scrapic) injected intracerebrally with either ME7 (scrapie) or normal (control) brain homogenate at each of the indicated time points. The activity was measured as the number of rectangles crossed over a 3-min period. The increase in motor activity in ME7-injected mice was replicated, but in this experiment, it occurred 2 weeks later on in the course of the disease (cf. Figure 5). It can be seen that there was a statistically significant increase in the activity of ME7-injected mice at 14, 18, and 20 weeks. The decline in motor activity of ME7-injected mice was seen from 20 weeks postinjection. ${ }^{*} p<.05, t$ test, two-tailed. N.B.: The number of scrapie mice is low because of fightinginduced mortality.

injection of ME7, our "targeting" of the hippocampus by using the ME7 strain of scrapie (Fraser \& Dickinson, 1973), or a combination of both factors. Whichever account is correct, it is clear that our method of inducing scrapie in the brain led to behavioral consequences that were detectable notably early in the course of the disease. The behavioral changes are also apparently selective in that different consequences develop after different latencies (open-field locomotor activity increased before the impairment of muscle function shown by the inverted-screen and horizontal-bar tests). This model offers a paradigm for investigating interventions that could slow the course of the disease, ameliorate its consequences, or both.

\section{Changes in Motor Activity}

Hippocampal lesions frequently produce locomotor hyperactivity, either directly or as a consequence of failure to habituate to an environment (Gray, 1982). We have shown that there is an increase in the activity of ME7injected mice from as early as 12 weeks postinjection (i.e., from approximately $50 \%$ of the way through the incubation period). The activity of the ME7-injected mice did not start to decline until 20-22 weeks postinjection (i.e., $83 \%-92 \%$ of the incubation period). Interestingly, the peak in locomotor activity in Experiment 3 occurred at a time ( 20 weeks) when the mice were showing significant impairment on the screen and bar tests of muscle strength and coordination. These findings are similar to those reported by McFarland and Hotchin (1980), who showed, in another mouse strain/scrapie strain combination that there was generalized hyperactivity in scrapieaffected mice from $60 \%$ of the way through the incubation period. This study did not comment on the level of activity seen in the later stages of the disease. A reduction in the activity of scrapie-affected mice was reported in the late stages of disease in another study (Hunter et al., 1986) that also used another mouse strain/scrapie strain combination. In complete contrast, an earlier study (Suckling et al., 1976) reported an "excitable phase" characterized by overactivity of scrapie-affected mice at $95 \%$ of the incubation period. In this study, earlier stages of the disease were said to be associated with a reduction in activity. Finally, one study did not reveal any change in motor activity in scrapie-affected mice (Savage \& Field, 1965). As noted, the variability between and within these studies may be attributable to factors such as the strain of mice and scrapie agent and/or duration of incubation.

In summary, it would appear that the activity of scrapieaffected mice depends on the details of the testing procedure, particularly on the route of injection. Making a focal injection into a structure whose dysfunction normally results in hyperactivity (i.e., hippocampus) should

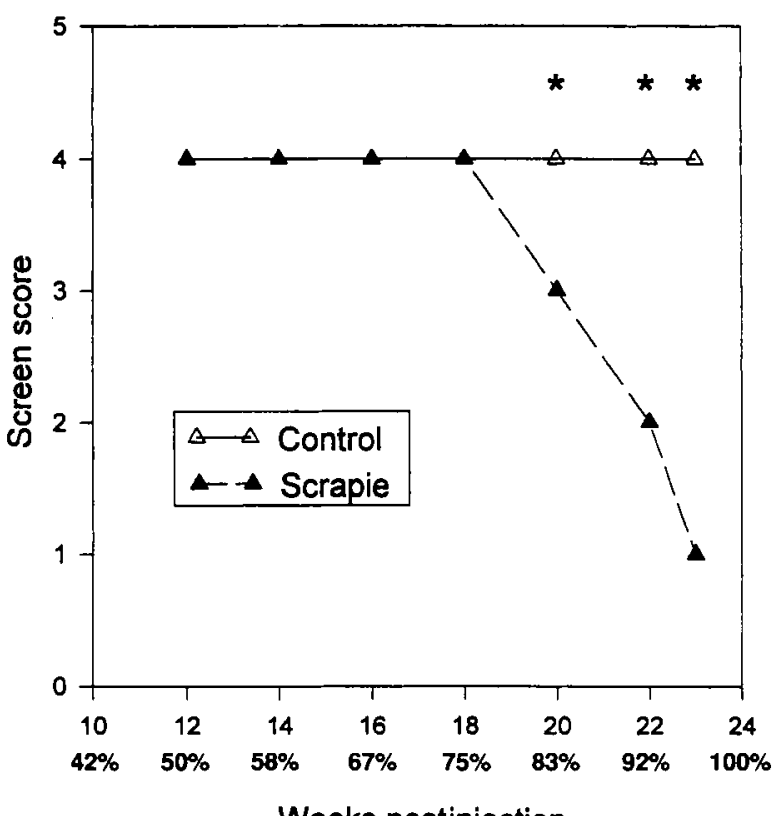

Weeks postinjection

Figure 9. Experiment 3: Median scores for the inverted-screen test in male mice ( $n=10$ control, 6 scrapie) injected with either ME7 (scrapie) or normal brain homogenate (control) at each of the indicated time points. There was a statistically significant reduction in muscle function from 20 weeks in ME7-injected mice, but not in control mice. ${ }^{\star} p<.05$, Mann-Whitney $U$ test, twotailed. N.B.: The number of scrapie mice is low because of fighting-induced mortality. 


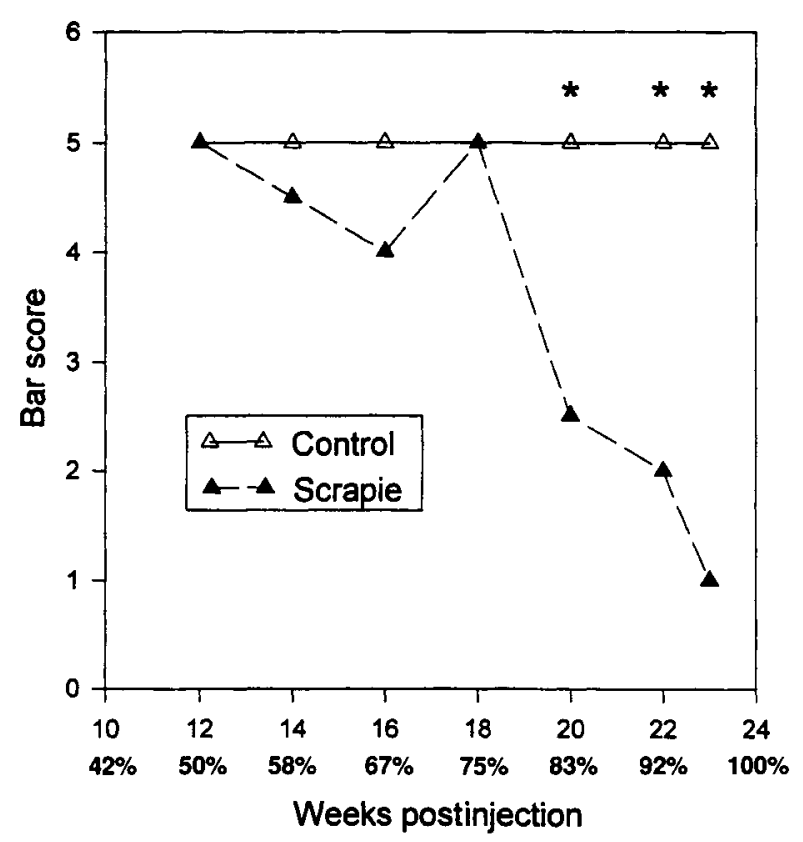

Figure 10. Experiment 3: Median scores for the horizontal-bar test in male mice ( $n=10$ controls, 6 scrapie) injected with either ME7 (scrapie) or normal brain homogenate (control) at each of the indicated time points. There was a statistically significant reduction in muscle function from 20 weeks in ME7-injected mice, but not in control mice. ${ }^{\star} p<.05$, Mann-Whitney $U$ test, two-tailed.

produce a more predictable change in activity than the ill-defined i.c. injection used hitherto. It is possible that different brain regions are preferentially, and sequentially, involved depending on the site of i.c. injection, and this may influence the profile of behavioral changes that is observed.

\section{Changes in Passive Avoidance}

Hippocampal lesions generally impair passive avoidance behavior, especially if there is an escape component, as in the present technique (Gray, 1982). The dosedependent impairment of passive avoidance acquisition in our scopolamine-treated mice confirmed the general sensitivity of the passive avoidance task. This impairment was similar to that seen in a previous study in another laboratory, which used a different apparatus and mouse strain (Deacon, 1990). Scopolamine frequently produces effects similar to those of hippocampal dysfunction (Dunnett, 1985), but it should be noted that Experiment 1 was not designed to be an exact template for the scrapie experiments; conventional hippocampal lesion before and after acquisition would be necessary for this.

The use of a multitrial rather than the more usual singletrial passive avoidance technique has several advantages: (1) The rate of acquisition can be directly assessed separately from retention, and (2) all animals are required to learn to a specific criterion rather than being subjected to a single trial, which may result in variable amounts of learning between different animals. Therefore, more meaningful results are obtained with fewer animals. The procedure used here (a single acquisition session followed by repeated testing for retention) also allows dissociation of treatment effects on footshock sensitivity (and/or learning) from effects on memory. Specifically, since each animal has to meet a set criterion at each test session, on the subsequent retention test, memory deficits would cause a greater proportion of avoidance failures within a group, whereas loss of footshock sensitivity or learning ability would result in an increase in trials to relearn only in those animals that failed to maintain passive avoidance behavior. A previous study by Hunter et al. (1986) showed that scrapie does not affect footshock sensitivity, even when clinical signs are evident. Subjective observation in our Experiments 2 and 3 showed that the shock sensitivity remained equal between groups; more recently we have developed a scoring system that could be used to quantify the reactivity of footshock in future work.

The early impairment in passive avoidance retention observed in ME7-injected mice might have been a direct result of the increased locomotor activity. This is unlikely, however; in a previous study amphetamine, at a dose $(2 \mathrm{mg} / \mathrm{kg}$ ) known to increase locomotion, significantly enhanced the rate of passive avoidance acquisition (Deacon, 1990). The avoidance failures during retention can be attributed to a dysfunction of either (1) the memory for or (2) the performance of the passive avoidance task.

The present observation of impaired passive avoidance in ME7-affected mice complements previous work by Hunter et al. (1986) but differs in some important respects. Hunter et al. used (1) CD-1 mice because learning in the C57BL strain was reported to be too variable, (2) a one-trial passive avoidance procedure without an escape component, (3) four different strains of scrapie agent, and (4) "low" and "high" shock levels ( 0.45 and $0.60 \mathrm{~mA}$, respectively). Impairments were seen only in mice affected with the 79A and 139A strains, and only at the lower shock level. It should be noted that, unlike our subjects, these mice were already in the early clinical stages of the disease, showing ataxia and abnormal posture or tremors. In contrast to the findings of our study, mice injected with the ME7 or $22 \mathrm{C}$ strain showed neither clinical signs nor passive avoidance impairment. Our route of injection and choice of behavioral parameters thus appears to yield important differences in the outcomes observed.

\section{Conclusion}

We have previously shown that there is an early inflammatory response in mouse scrapie that precedes the development of neuronal loss and the appearance of typical clinical signs of scrapie (Betmouni et al., 1996). This inflammatory response is likely to be an indicator of some as yet uncharacterized pathology, but it may arise in response to the deposition of PrPsc. We have now shown that in our focal injection paradigm, it is possible 
to detect more subtle clinical signs earlier during the course of disease by using behavioral tasks that may be affected by lesions of the hippocampus. The passive avoidance task deficit and the hyperactivity observed early in the course of the disease may reflect dysfunction of hippocampal neurons. Studies are now in progress to examine the effects of i.c. injection of scrapie agent in the rat, a species that will allow fuller investigation of cognitive function.

It is possible that the inflammatory response may contribute to changes in neuronal function detectable behaviorally by an alteration in activity and passive avoidance tasks. At this stage of the work, we do not know how closely these early behavioral changes relate to the inflammatory response. Future studies will need to address this question.

The investigation of early histopathological and clinical changes in mouse scrapie is important not only in relation to the prion diseases but also in relation to other, more common, chronic neurodegenerative conditions such as Alzheimer's disease. An understanding of the events that precede irreversible neuronal loss in a slowly evolving disease of the central nervous system is key to the elucidation of the mechanisms underlying chronic neurodegeneration, as well as to any potential therapeutic strategies whose efficacy may depend on whether or not neuronal loss is established.

\section{REFERENCES}

Betmouni, S., Perry, V. H., \& Gordon, J. L. (1996). Evidence for an early inflammatory response in the central nervous system of mice with scrapie. Neuroscience, 74, 1-5.

DEACON, R. M. J. (1990). Benzodiazepine receptor inverse agonists improve acquisition of a multi-trial passive avoidance task. British Journal of Pharmacology, 100, 423P.

DEACON, R. M. J. (1998). Unpublished raw data.

DunNetT, S. B. (1985). Comparative effects of cholinergic drugs and lesions of the nucleus basalis or fimbria-fornix on delayed matching in rats. Psychopharmacology, 87, 357-363.

Fraser, H., \& Dickinson, A. G. (1973). Scrapie in mice: Agent-strain differences in the distribution and intensity of grey matter vacuolation. Journal of Comparative Pathology, 83, 29-40.
GraY, J. A. (1982). The neuropsychology of anxiety: An enquiry into the functions of the septo-hippocampal system (pp. 115-183). Oxford: Oxford University Press.

Gray, J. A., \& MCNaUghton, N. (1983). Comparison between the behavioural effects of septal and hippocampal lesions: A review. Neuroscience \& Behavioral Reviews, 7, 119-188.

Hadlow, W. J., KenNedy, R. C., \& RaCe, R. E. (1982). Natural infection of Suffolk sheep with scrapie virus. Journal of Infectious Diseases, 146, 657-664.

Heitzman, R. J., \& CoRP, C. R. (1968). Behaviour in emergence and open-field tests of normal and scrapie mice. Research in Veterinary Science, 9, 600-601.

Hunter, A. J., Caulfield, M. P., \& Kimberlin, R. H. (1986). Learning ability of mice infected with different strains of scrapie. Physiology \& Behavior, 36, 1089-1092.

KREUTZBERG, G. W. (1996). Microglia: A sensor for pathological events in the CNS. Trends in Neurosciences, 19, 312-318.

Lawson, L. J., Perry, V. H., Dri, P., \& Gordon, S. (1990). Heterogeneity in the distribution and morphology of microglia in normal adult and mouse brain. Neuroscience, 39, 151-170.

LySONS, A. M., \& WoOLlaRd, S. J. (1996). Spatial reversal learning in preclinical scrapie-inoculated mice. NeuroReport, 7, 1087-1091.

MCFARLAND, D., \& HotCHIN, J. (1980). Early behavioural abnormalities on mice due to scrapie virus encephalopathy. Biological Psychiatry, $15,37-44$.

Outram, G. W. (1972). Changes in drinking and feeding habits of mice with experimental scrapie. Journal of Comparative Pathology, 82, 412-427.

Prusiner, S. B. (1982). Novel proteinaceous infectious particles cause scrapie. Science, 216, 136-144.

Prusiner, S. B. (1996). Molecular biology and pathogenesis of prion diseases. Trends in Biological Sciences, 21, 482-487.

RabinowitZ, S. S., \& GoRDON, S. (1991). Macrosialin, a macrophage restricted membrane sialoprotein differentially glycosylated in response to inflammatory stimuli. Journal of Experimental Medicine, 174, 827-836.

Savage, R. D., \& Field, E. J. (1965). Brain damage and emotional behaviour: The effects of scrapie on emotional responses of mice. Animal Behaviour, 13, 443-446.

Suckling, A. J., Bateman, S., Waldron, C. B., Webb, H. E., \& KimBERLIN, R. H. (1976). Motor activity changes in scrapie-affected mice. Journal of Experimental Pathology, 57, 742-746.

TOMONARI, K. (1988). A rat antibody against a structure functionally related to the mouse T-cell receptor/T3 complex. Immunogenetics, 28, 455-458.

(Manuscript received April 17, 1998; revision accepted for publication September 16, 1998.) 